Article

\title{
Human Endometrial Carcinogenesis Is Associated with Significant Reduction in Long Non-Coding RNA, TERRA
}

\author{
Meera Adishesh ${ }^{1,2}$, Rafah Alnafakh ${ }^{2}$, Duncan M. Baird ${ }^{3}{ }^{(0}$, Rhiannon E. Jones ${ }^{3}$, \\ Shannon Simon ${ }^{2}$, Lucy Button ${ }^{2}$, Areege M. Kamal ${ }^{2,4}{ }^{\circledR}$, John Kirwan ${ }^{1}$, S. Bridget DeCruze ${ }^{1}$, \\ Josephine Drury ${ }^{2} \mathbb{D}$, Gabriele Saretzki ${ }^{5}$ and Dharani K. Hapangama ${ }^{1,2, *(\mathbb{D}}$ \\ 1 Liverpool Women's Hospital NHS Foundation Trust, Liverpool L8 7SS, UK; \\ meeadish@liverpool.ac.uk (M.A.); John.Kirwan@lwh.nhs.uk (J.K.); shandyabdecruze@gmail.com (S.B.D.) \\ 2 Department of Women's and Children's Health, Institute of Life Course and Medical Sciences, \\ University of Liverpool, Member of Liverpool Health Partners, Liverpool L8 7SS, UK; \\ R.A.A.Alnafakh@liverpool.ac.uk (R.A.); shannonsimon96@gmail.com (S.S.); lucyfbutton@gmail.com (L.B.); \\ areegekamal@gmail.com (A.M.K.); jadrury@liverpool.ac.uk (J.D.) \\ 3 Division of Cancer and Genetics, Cardiff University, Cardiff CF14 4XN, UK; bairddm@cardiff.ac.uk (D.M.B.); \\ jonesr47@cardiff.ac.uk (R.E.J.) \\ 4 The National Centre for Early Detection of Cancer, Oncology Teaching Hospital, Baghdad Medical City, \\ Baghdad, Iraq \\ 5 Biosciences Institute, Newcastle University, Newcastle upon Tyne NE4 5PL, UK; \\ gabriele.saretzki@newcastle.ac.uk \\ * Correspondence: dharani@liv.ac.uk; Tel.: +44-151-795-9553
}

Received: 3 November 2020; Accepted: 16 November 2020; Published: 18 November 2020

\begin{abstract}
Telomeres are transcribed as long non-coding RNAs called TERRAs (Telomeric repeat containing RNA) that participate in a variety of cellular regulatory functions. High telomerase activity (TA) is associated with endometrial cancer (EC). This study aimed to examine the levels of three TERRAs, transcribed at chromosomes 1q-2q-4q-10q-13q-22q, 16p and 20q in healthy $(n=23)$ and pathological $(n=24)$ human endometrium and to examine their association with cellular proliferation, TA and telomere lengths. EC samples demonstrated significantly reduced levels of TERRAs for Chromosome 16p (Ch-16p) ( $p<0.002)$ and Chromosome 20q (Ch-20q) $(p=0.0006)$, when compared with the postmenopausal samples. No significant correlation was found between TERRA levels and TA but both Ch-16p and Ch-20q TERRA levels negatively correlated with the proliferative marker Ki67 ( $r=-0.35, p=0.03$ and $r=-0.42, p=0.01$ respectively). Evaluation of single telomere length analysis (STELA) at XpYp telomeres demonstrated a significant shortening in EC samples when compared with healthy tissues $(p=0.002)$. We detected TERRAs in healthy human endometrium and observed altered individual TERRA-specific levels in malignant endometrium. The negative correlation of TERRAs with cellular proliferation along with their significant reduction in EC may suggest a role for TERRAs in carcinogenesis and thus future research should explore TERRAs as potential therapeutic targets in EC.
\end{abstract}

Keywords: TERRA; long non-coding RNA; telomerase; endometrium; telomeres; telomere length; endometrial cancer

\section{Introduction}

Endometrial cancer (EC) is the most common gynecological malignancy in the western world with an increasing incidence due to the rise in obesity and longevity [1]. This increase in frequency of EC is 
accompanied by a concomitant rise in cancer-associated mortality, a trend that is expected to continue for the next few decades [2]. This is a contrasting and concerning statistic, compared with many other cancers, where the incidence of new cancers remains stable and cancer-associated mortality is decreasing. Therefore, it poses an urgent need to develop novel preventative and treatment strategies, which require advancement in our current understanding of endometrial carcinogenesis. The mortality rates for high-grade EC are especially high, similar to ovarian cancer. Some women with EC are not suitable for the conventional surgical treatment, hysterectomy [3], and surgical treatment is associated with significant morbidity and mortality in those with co-occurring multi-morbidities and obesity. Biomarkers that would inform us of potential prognostic and therapeutic targets are thus invaluable in developing future strategies to reduce the cancer related morbidity and mortality of EC.

High activity levels of telomerase enzyme are a feature of most carcinomas, with telomere maintenance being its main function. Telomeres are specialized nucleoprotein complexes at the ends of chromosomes that prevent chromosomal ends from being recognized as DNA damage foci that can mount a DNA damage response (DDR) [4]. Telomeres contain a repetitive hexanucleotide sequence TTAGGG bound by six-shelterin proteins, namely telomeric repeat binding factor 1 and 2 (TRF1 and TRF2), Protection of telomeres 1 (POT1), tripeptidyl peptidase 1 (TPP1), Repressor/activator protein 1 (RAP1) and TRF1- and TRF1-interacting protein 2 (TIN2), and ends with single-stranded G-rich overhang [5]. Telomeric DNA is lost with each round of cell division, due to the end replication problem, and, unless the lost DNA is replaced by the action of telomerase, telomeres will shorten in proliferating cells. Critically short telomeres can initiate a DDR and induce apoptotic or cellular senescence pathways with permanent cell cycle arrest [6]. However, specialized cells and cancer cells have sufficiently high telomerase activity (TA) for telomere maintenance and elongation, which prevents senescence and allows unrestrained proliferation [7].

Telomeres were considered to be transcriptionally silent until recently, however telomeric repeat-containing RNA (TERRA) molecules are transcribed by RNA polymerase II from CpG-island containing sub telomeric promotors [8]. TERRA molecules are heterogenous long non-coding RNAs (lncRNA) [9], and they have been identified in vertebrates and in several other species including yeasts and plants $[10,11]$. Transcription of TERRA has been proposed to assist in telomere replication and thus support chromosome stability [12]. The action of TERRA may be relevant to the telomere of origin or other telomeres in general [13]. Their expression is inversely correlated with the length of the corresponding telomeres [14]. TERRAs are involved in the recruitment of telomerase to telomeres [15] when they remain partly associated with the telomere of origin, establishing an RNA G-quadruplex structure that protects the telomeres [16]. The TERRA containing RNA-DNA hybrids at the telomeric end may also have a physiological role in stalling replication forks, hence allowing DNA repair [17]. Conversely, TERRAs are also proposed to inhibit telomerase function by binding to the hTERC and hTERT components of telomerase [18], as well as by forming RNA-DNA hybrids [10]. TERRA levels are cell specific [9] and fluctuate with the cell cycle [19]. Shelterin proteins TRF1 and TRF2 are known to interact with TERRA and TERRAs mediate cell-fate and cellular reprogramming via a TRF1-dependent pathway [20]. Therefore, TERRAs are thought to participate in many essential cellular and telomere/telomerase regulatory functions [14]. A study that examined the predicted features of 18 TERRA loci in human cell confirmed transcripts arising from only 20q and Xp loci to have these expected TERRA features, and subsequently demonstrated that only deletion of the 20q locus to cause a dramatic decrease in TERRA levels [13]. By deleting the 20q-TERRA locus, the authors demonstrated an increase in telomere damage foci and confirmed that TERRA transcripts to be essential for the maintenance of a functional telomere cap.

Human endometrium is a dynamic tissue, which undergoes a repetitive cycle of cell proliferation, differentiation, shedding and regeneration during the reproductive years of a woman's life. It is regulated by the cyclically secreted ovarian hormones on a monthly basis [21]. Although a somatic tissue, human endometrium expresses dynamic TA levels, and highly proliferative endometrial cells during the estrogen dominant, proliferative phase of the menstrual cycle have high TA levels, while the 
cessation of the epithelial proliferation under the regulation of progesterone in the secretory phase of the cycle demonstrated lowest TA levels [4,22]. Endometrial epithelium demonstrates distinctively higher TA than the stromal cells, yet conversely shorter relative telomere lengths were reported in the epithelial cells when compared with the stromal cells from the same samples [22]. This observation had been explained as high TA maintaining the short epithelial telomeres beyond the critical length, thus allowing epithelial cells to avoid cell cycle arrest/cellular senescence [4,22]. Therefore, TA is implicated in endometrial epithelial cell proliferation; high TA is a reported feature in the normal proliferative phase endometrium, in benign endometrial proliferative conditions such as endometriosis, and in endometrial carcinogenesis [23,24].

As mentioned above, many authors have already reported the involvement of TA, telomeres and many telomere/telomerase associated proteins and genes in endometrial function including their aberrations in endometrial carcinogenesis [25-30]. However, the existence of TERRAs in healthy endometrium or their disease-specific alterations have not yet been elucidated. Considering the intricate relationship between telomere/telomerase biology and endometrial cellular proliferation [22] and carcinogenesis [31], we hypothesized that TERRA levels may also be altered in EC. We therefore sought to examine the expression levels of TERRAs in human endometrium, from both healthy women and those with EC. We utilized TERRA primers that measured collective TERRA levels from various chromosome ends (chromosomes 1q, 2q, 4q, 10q, 13q and 22q) and two further specific TERRAs that are transcribed from chromosomes 16p and 20q.

We also examined the relationship of TERRA levels with epithelial cell proliferative index and steroid hormone receptors immuno-scores assessed using immunohistochemistry, as they are established markers predicting prognosis in EC. We also examined the immunoreactivity of two shelterin proteins known to regulate TERRAs, TRF1 (positive regulator) and TRF2 (negative regulator), in the same EC samples to examine their possible correlation with the corresponding TERRA levels. Finally, we analyzed the differential telomere length distributions using high-resolution single telomere length analysis (STELA) at the chromosome XpYp telomeres (which is representative of the genome-wide telomere length) in the same human endometrial samples.

\section{Results}

\subsection{TERRA Transcription Is Observed in Normal Human Endometrium and the Levels Are Significantly Reduced in Endometrial Cancer}

Both pre- and postmenopausal endometrium expressed all TERRAs examined (chromosomes 1q-2q-4q-10q-13q-22q, 16p and 20q) (Figure 1A-C). Compared with the proliferative phase samples, the proliferatively quiescent postmenopausal samples had higher levels of Ch-16p and Ch-20q TERRA levels (Figure 1B,C).

Ch-16p $(p=0.002)$ and Ch-20q ( $p=0.0006)$ TERRAs were significantly reduced in EC samples when compared with the healthy postmenopausal endometrium (Figure 1B,C). However, TA was high in proliferative phase and EC samples ( $p=0.19$, Figure 1D). In our sample set, the only significant difference in TA was found between the proliferative and secretory phase samples from pre-menopausal women $(p=0.04)$ as previously described [4,32].

When the histological grading (as a measure of cellular anaplasia) of EC samples or individual subtypes of type II ECs were considered, neither TERRA levels nor TA changed significantly with the tumor grade/subtype (Figure 2). 
A

TERRA 1q-2q-4q-10q-13q-22q

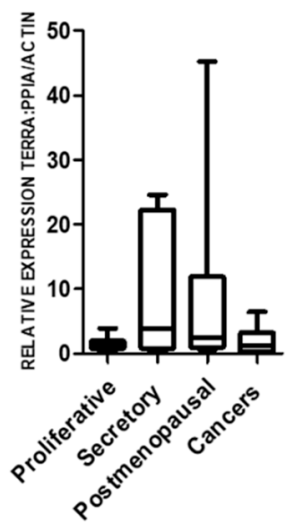

C

TERRA 20q

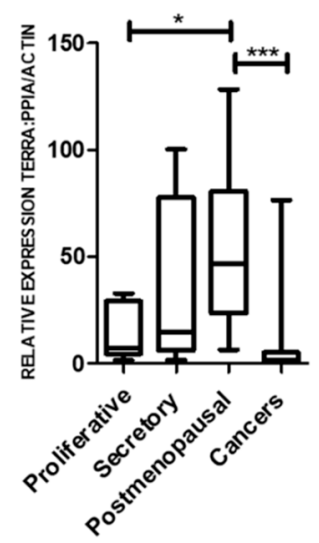

B

TERRA 16p

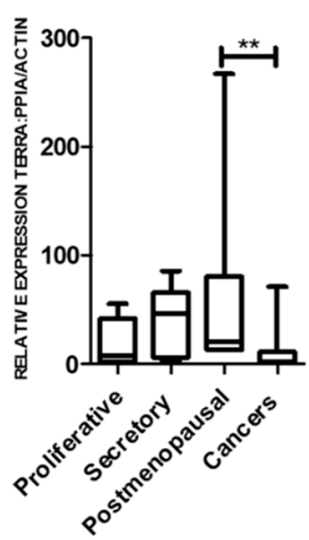

D

TRAP

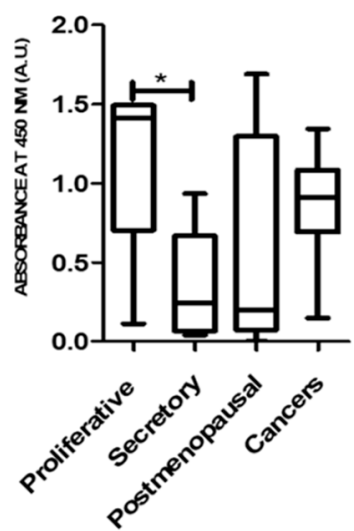

Figure 1. TERRA levels in human endometrium. TERRA levels by qPCR and Telomerase activity by Telomere Repeat Amplification Protocol (TRAP) assay in proliferative $(n=6)$, secretory $(n=8)$, postmenopausal $(n=7)$ human endometrium and endometrial cancers $(n=24)$ for: chromosome 1q-2q-4q-10q-13q-22q (A); chromosome 16p (B); and chromosome 20q (C). When compared to age-matched postmenopausal endometrium $(n=7)$, women with ECs $(n=24)$ showed no changes in TERRA levels for chromosomes 1q-2q-4q-10q-13q-22q (A) but a significant decrease in Ch-16p (** $p=<0.002$ ), Mann-Whitney-U test (B) and Ch-20q ( $\left.{ }^{* * *} p=<0.001,{ }^{*} p<0.05\right)$; Mann-Whitney-U test (C) but no changes in TA (D). 
A

TERRA $1 q-2 q-4 q-10 q-13 q-22 q$

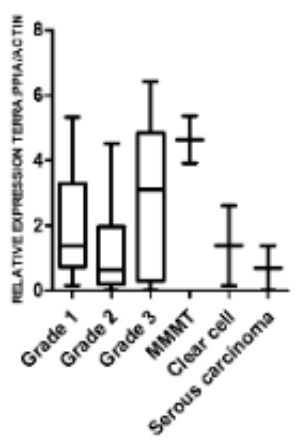

C

TERRA 20q

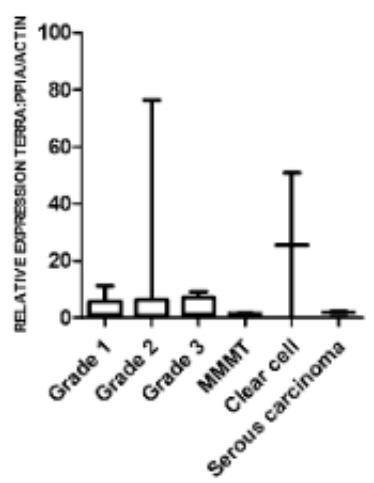

B

TERRA 16p

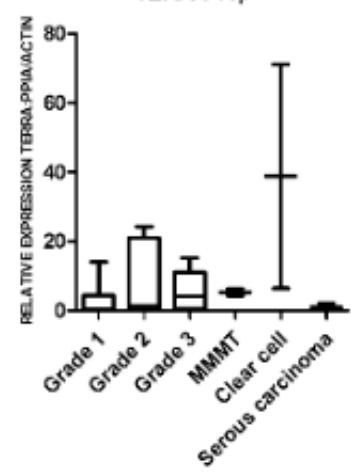

D

TRAP

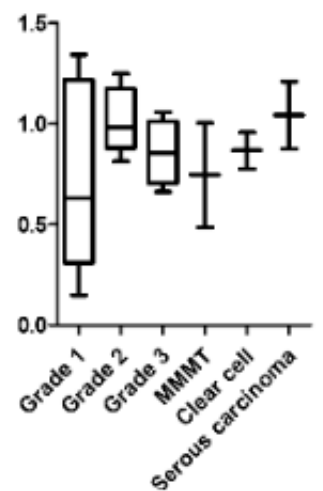

Figure 2. TERRA and TRAP levels in different grades and types of endometrial cancers. Grade 1 adenocarcinoma, $n=6$; grade 2 adenocarcinoma, $n=7$; grade 3 adenocarcinoma, $n=5$; carcinosarcoma/malignant mixed Müllerian tumor (MMMT), $n=2$; clear cell carcinoma, $n=2$; serous carcinoma, $n=2$. Relative normalized expression of chromosomes: $1 \mathrm{q}-2 \mathrm{q}-4 \mathrm{q}-10 \mathrm{q}-13 \mathrm{q}-22 \mathrm{q}(\mathrm{A})$; $16 \mathrm{p}(\mathbf{B})$; and 20q (C). TERRA levels were measured in all types of endometrial cancers. No difference in TERRA levels were found in different grades of endometrioid cancers or individual subtypes of type 2 cancers. All types of endometrial cancers had high telomerase activity as assessed by TRAP assay (D).

2.2. Endometrial TERRA Levels Correlated with Each Other, the Proliferative Marker Ki67, Steroid Receptor PR and Shelterin Protein TRF1 but Did Not Correlate with TA or with TLs at the XpYp Chromosomes

Levels of all three TERRAs demonstrated a positive correlation with each other in all endometrial samples (healthy and pathological) examined, suggesting a shared regulation (Table 1: Ch-16p and 20q, $\mathrm{r}=0.78, p<0.0001$; Ch-16p and Ch-1q-2q-4q-10q-13q-22q, r = 0.49, $p=0.001$; Ch-20q and Ch-1q-2q-4q-10q-13q-22q, r = 0.48, $p=0.001)$. However, TERRA levels did not correlate with TA. Ki-67 proliferative indices correlated negatively with Ch-16p and Ch-20q TERRAs (Table 1: $\mathrm{r}=-0.35$, $p=0.03 ; \mathrm{r}=-0.42, p=0.01$ respectively) suggesting a role related to cell proliferation. Ki67 scores did not correlate with Ch-1q-2q-4q-10q-13q-22q. Ch-16p TERRA also correlated negatively with the Liverpool endometrial steroid quick score (LESQS) for PR ( $r=-0.40, p=0.02)$ but the LESQS for other hormone receptors did not show a significant association with TERRA levels (Table 1 and Figure 3). The publicly available "The Cancer Genome Atlas" (TCGA) cohort of uterine cancers dataset suggests high expression of TRF1 gene (TERF1 RNA) in ECs to be associated with decreased survival (Figure S1). Immunostaining quick-scores for TRF1 protein levels correlated positively with Ch-20q TERRA levels ( $\mathrm{r}=0.71, p=0.001$, Table 1$)$. 
Table 1. Correlation of chromosomes 1q-2q-4q-10q-13q-22q (TERRA 1), TERRA 16 TERRA 20, LESQS scores for AR, PR, ER $\alpha$ and ER $\beta$ and immuno-scores for Ki67, TRF1, TRF2 and TA measured by TRAP assay in all endometrial samples. Values in bold letters show significant correlations

\begin{tabular}{ccccccc}
\hline & TERRA1 & \multicolumn{3}{c}{ TERRA16 } & \multicolumn{3}{c}{ TERRA20 } \\
\hline & $\mathbf{r}$ & $\boldsymbol{p}$ & $\mathbf{r}$ & $\boldsymbol{p}$ & $\mathbf{r}$ & $\boldsymbol{p}$ \\
\hline TERRA1 & & & $\mathbf{0 . 4 8 8 5}$ & $\mathbf{0 . 0 0 0 8}$ & $\mathbf{0 . 4 8 4 1}$ & $\mathbf{0 . 0 0 0 9}$ \\
\hline TERRA16 & $\mathbf{0 . 4 8 8 5}$ & $\mathbf{0 . 0 0 0 8}$ & & & $\mathbf{0 . 7 7 9 2}$ & $<\mathbf{0 . 0 0 0 1}$ \\
\hline TERRA20 & $\mathbf{0 . 4 8 4 1}$ & $\mathbf{0 . 0 0 0 9}$ & $\mathbf{0 . 7 7 9 2}$ & $<\mathbf{0 . 0 0 0 1}$ & & \\
\hline AR & -0.08156 & 0.6465 & -0.2828 & 0.1051 & -0.1727 & 0.3288 \\
\hline PR & -0.1068 & 0.5543 & $\mathbf{- 0 . 3 9 9 7}$ & $\mathbf{0 . 0 2 1 2}$ & -0.2094 & 0.2423 \\
\hline ER $\boldsymbol{\alpha}$ & -0.0579 & 0.7411 & 0.1462 & 0.4019 & 0.198 & 0.2543 \\
\hline ER $\boldsymbol{\beta}$ & 0.01555 & 0.9293 & 0.1325 & 0.4479 & 0.08311 & 0.635 \\
\hline Ki67 & -0.147 & 0.3854 & $\mathbf{- 0 . 3 4 8 7}$ & $\mathbf{0 . 0 3 1 9}$ & $\mathbf{- 0 . 4 1 5 6}$ & $\mathbf{0 . 0 0 9 5}$ \\
\hline TRF1 & 0.4547 & 0.0505 & 0.4414 & 0.0585 & $\mathbf{0 . 7 0 8 3}$ & $\mathbf{0 . 0 0 0 7}$ \\
\hline TRF2 & 0.08588 & 0.8432 & 0.4269 & 0.2499 & 0.4809 & 0.1938 \\
\hline TRAP & -0.1997 & 0.2292 & -0.251 & 0.1285 & -0.09328 & 0.5775 \\
\hline
\end{tabular}

(A) ER $\alpha$ in EC

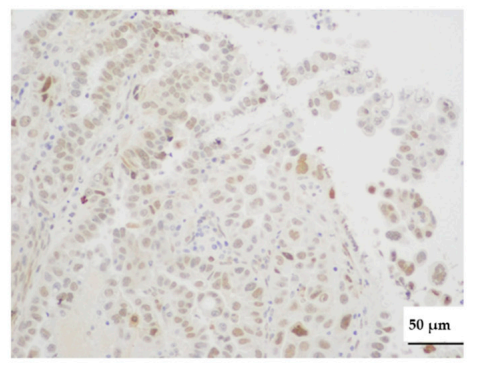

(C) PR in EC

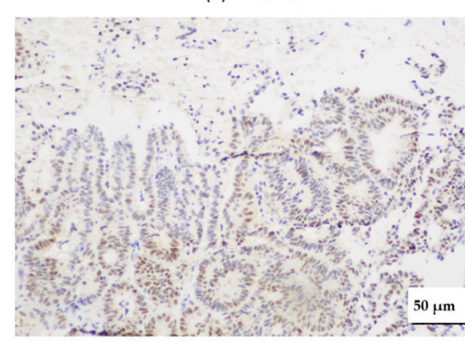

(E) Ki67 in PM endometrium

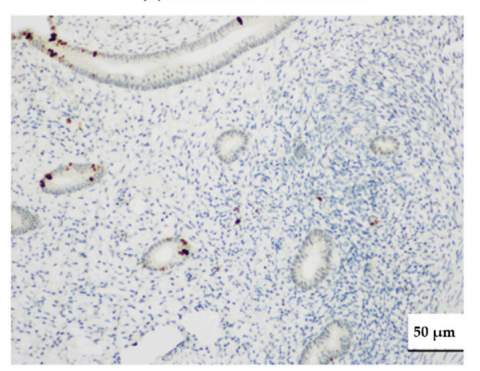

(B) $E R \beta$ in $E C$

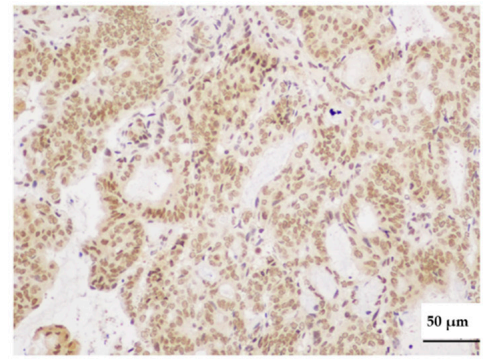

(D) AR in EC

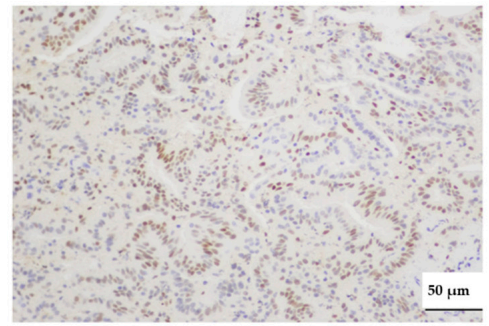

(F) Ki67 in EC

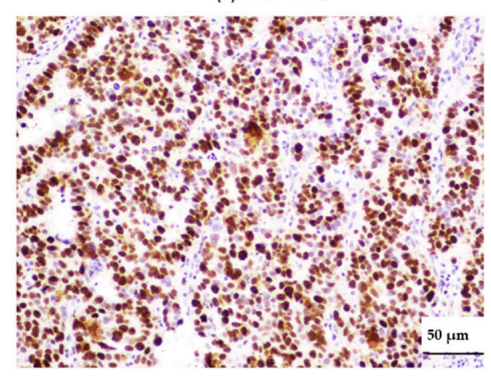

Figure 3. Representative micrographs of steroid receptors and Ki67. Micrograph showing immunostaining in endometrial cancer $(E C)$ of: estrogen receptor $\alpha(E R \alpha)(A)$; estrogen receptor $\beta$ (ER $\beta)$ (B); progesterone receptor (PR) (C); and androgen receptor (AR) (D). Micrographs of Ki67 immunostaining: in postmenopausal (PM) endometrium (E); and in endometrial cancer (F). Scale bar $50 \mu \mathrm{m}, 200 \times$ magnification. 
Clear differences were observed between healthy postmenopausal and EC samples in terms of both telomere lengths and variance (SD) of the distribution at the XpYp chromosomes (which is representative of the genome-wide telomere length) (Figure 4A). Postmenopausal endometrial samples displayed considerable telomere length heterogeneity with telomeres at XpYp chromosomes ranging in one sample from 1.3 to $21.7 \mathrm{~kb}$. Consistent with previous reports (reviewed in [5]), ECs displayed shorter telomeres when compared with healthy postmenopausal endometrium ( $p=0.002$, Figure 4B). We found a trend towards a decrease of telomere lengths in older women; however, this difference was not significant (Spearman correlation $r=-0.49, p=0.09$, Figure 4C).

A

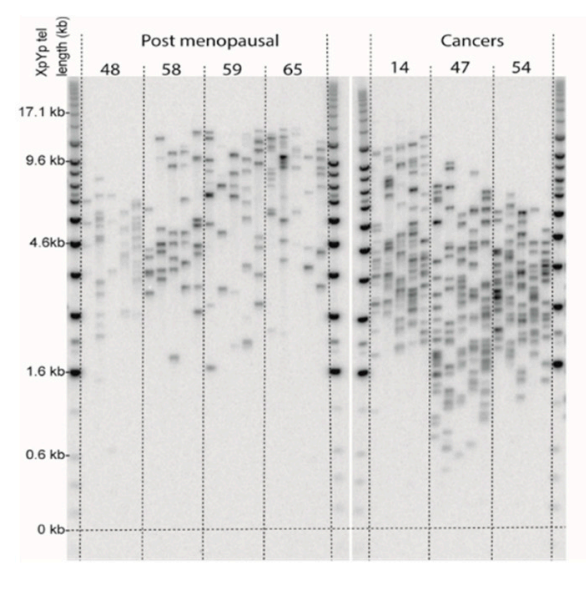

B

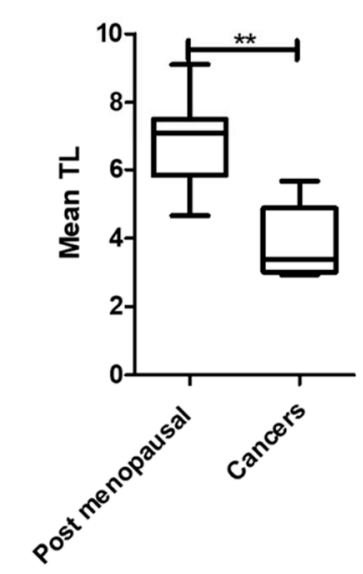

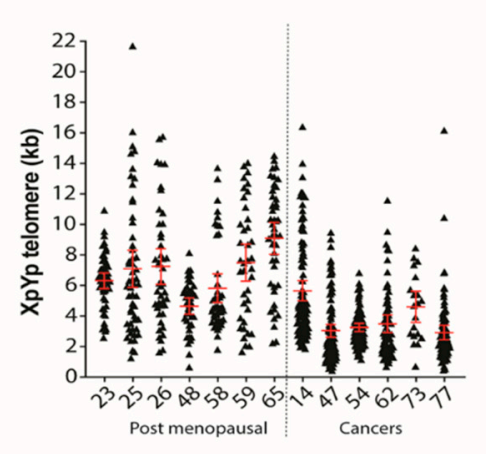

C

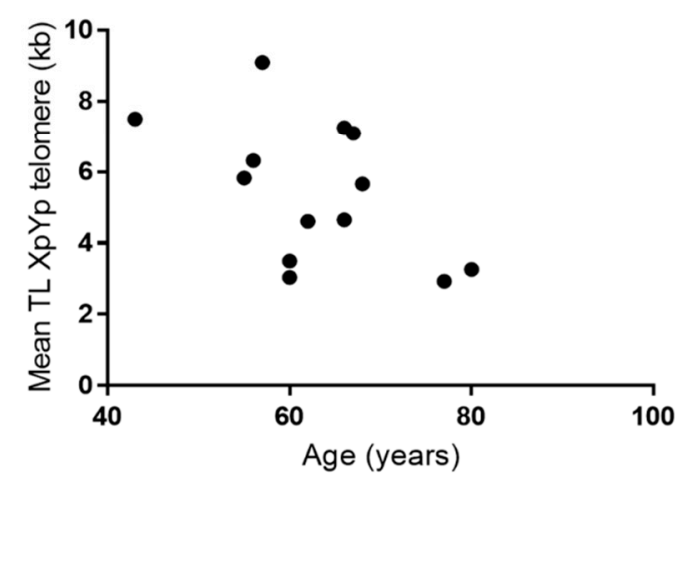

Figure 4. Single telomere length analysis at XpYp telomeres, telomere length distributions in healthy post-menopausal endometrial and endometrial cancer tissue. Representative STELA gel image, together with the distribution of telomere lengths represented as a scatter plot (A). Mean telomere lengths in healthy postmenopausal endometrium compared to endometrial cancers. Telomere lengths are significantly shorter in endometrial cancers compared to postmenopausal endometrium $(* * p=0.002$, Mann-Whitney-U test) (B). Telomere length correlated with age of the patients, demonstrating no significant association with age and mean telomere lengths (Spearman correlation, $\mathrm{r}=-0.49, p=0.09)(\mathbf{C}$ ).

\section{Discussion}

We report here the long non-coding RNA, TERRA, levels in healthy human endometrium from preand postmenopausal women and their significant reduction in expression in EC. Although TERRAs have been previously described in human cancers, to our knowledge, the evaluation of TERRA levels in endometrial cancers and their comparison with healthy human endometrial tissues has not been 
previously reported. The levels of different TERRAs are described in the context of TA, telomere lengths at chromosomes XpYp, immunoscores for ovarian hormone receptors, levels of shelterin proteins TRF1/2 and proliferative indices from the same patient-derived endometrial samples.

We employed a qPCR-based assessment of TERRA levels for its superior quantification quality and reproducibility. Human endometrium is a unique somatic tissue demonstrating dynamic TA, which regulates epithelial proliferation [4]. When we considered the highly proliferative epithelial cells in the proliferative phase endometrium from premenopausal women, which has been associated with high TA and relatively longer average telomere length [22], TERRA levels were remarkably low. The inverse correlation of TERRA levels with epithelial proliferative indices we observed further suggests an association with cellular quiescence for TERRAs in the human endometrium.

Each TERRA had a unique expression pattern, suggesting chromosome-specific regulation of TERRAs. Non-replicative cells with shorter telomeres undergo sub-telomeric rearrangements in yeasts during the quiescent phase of the cell cycle [33], and this further results in increased transcription of non-coding TERRA, agreeing with our data for Ch-20q TERRA in human tissue.

TRF1 and TRF2 are shelterin proteins which protect the chromosomal ends from fusion and initiation of a DDR and they help to regulate TA through a negative feedback mechanism [34]. TRF1 may provide a telomere stabilization function to the short telomeres that we and others have shown in ECs [5]. This may prevent arrest of the cell cycle, thus allowing EC cells to proliferate continuously. The publicly available "The Cancer Genome Atlas" (TCGA) cohort of uterine cancers dataset suggests high expression of TRF1 in ECs to be associated with decreased patient survival (Figure S1). Furthermore, TERRAs can bind to chromosomal locations other than telomeres [35], while the interaction between TERRA and TRF1/2 ensures that TERRA transcripts remain tethered to the telomeric domains [36]. We observed TRF1 protein levels to correlate positively with Ch-20q TERRA levels in EC samples. In contrast, the opposite was observed in benign mouse embryonic stem cells, where an increase in TERRA transcription was observed following abrogation of TRF1 and this may suggest a potential differential interaction between TRF1 and Ch-20q TERRA that may be specific to either human cancers or to EC [20].

We observed high TERRA levels in the proliferatively quiescent PM endometrium, which has low TA levels. In fission yeasts, upregulation of telomere-engaged TERRA can support telomerase-independent telomere maintenance [37]. The high TERRA levels that we report in the TA deficient healthy postmenopausal human endometrium may suggest similar function for human TERRAs, in sustaining telomeres in PM endometrium, preventing genetic instability. A recent in silico study, which examined the TCGA uterine cancers dataset, identified many critical genes associated with telomere maintenance that were previously unknown to contribute to endometrial carcinogenesis and prognosis [25]. However, the intricate relationship between these telomere and telomerase associated genes and proteins with TERRAs is yet to be fully elucidated. Considering the interesting and significant reduction in TERRA levels in EC samples we observed, further examination of this potential interaction is warranted in future studies.

A recent study analyzing endometrioid and serous cancer samples from the TCGA dataset reported EC to be one of the human cancers with the shortest mean telomere lengths among 31 different cancer types [38]. Our data examining individual telomere lengths using STELA at Chromosomes XpYp (which is representative of the genome-wide telomere length) [39] also demonstrate significantly shorter telomeres in EC. Although we were not able to assess the specific telomere lengths at the chromosomes corresponding to the TERRAs tested (Ch16 and 20), collectively, the above data suggest EC to have shorter telomeres. The complex mechanism of TERRA's role in regulation of telomere length has been previously reviewed and variable mechanisms involving different pathways have been proposed in different physiological conditions. For example, TERRA binding of TLS to G-quadruplex leading to accumulation of H4K20 trimethylation and TERRAs associating with LSD1 and MRE 11 in telomeric 3' $G$ overhang removal leading to lack of protection to chromosomal ends from being recognized as sites of DNA damage, therefore resulting in telomere shortening, were reported [40-43]. However, our data are the first to evaluate the comparative TERRA levels in healthy and malignant human endometrial tissue, 
thus we hypothesize that concurrent reduction in TERRA levels and telomere lengths observed in EC may be a global EC related phenomenon. At least, before telomerase re-activation, shorter telomeres may facilitate EC progression due to telomere dysfunction by initiating a "telomere crisis" that drives genomic instability and clonal evolution [44]. We therefore present this interesting observation of reduced TERRA levels in ECs, which co-exist with high TA and short telomeres, thus suggesting a complex, cancer-specific telomere biology in the endometrium. Furthermore, considering the proposed prevention of telomerase accessing telomeres by TERRA [40], the reduction of TERRA may provide a telomere maintenance function and thus proliferative advantage for the ECs. This aspect warrants further studies to ascertain the functional role of TERRA in the endometrium and in EC.

\section{Materials and Methods}

\subsection{Endometrial Tissue Samples}

Endometrial biopsies were obtained from 47 women undergoing gynecological surgery at Liverpool Women's Hospital. Ethical approval was obtained from the Liverpool Adult Local Research Ethics Committee (LREC; 09/H1005/55, 9 October 2009, NRES Committee North West-Liverpool Central, and 11/H1005/4 6 April 2011, NRES Committee North West-Liverpool Central) and informed written consent was obtained from all patients. Control endometrial samples were collected from women who were not on any hormonal treatment for at least 3 months, with no known endometrial pathology and with regular periods in proliferative phase $(n=7)$, secretory phase $(n=9)$ and postmenopausal $(n=7)$ women, as well as EC samples donated by 24 women with a prior diagnosis of EC (Endometrioid (grade $1 n=6$, grade $2 n=7$ and grade $3 n=5$ ) and 6 type 2 ECs (serous $n=2$, carcinosarcoma/malignant mixed Müllerian tumor (MMMT) $n=2$ and clear cell $n=2)$ ) undergoing hysterectomy without receiving any pre-surgical treatment. Patient clinico-pathological and demographic details were retrieved from the clinical notes and electronic databases (Table 2).

Table 2. Demographic features of study groups.

\begin{tabular}{ccc}
\hline Study Groups $(\boldsymbol{n})$ & ${ }^{*}$ Age (years) & ${ }^{*}$ BMI $\left(\mathbf{k g} / \mathbf{m}^{\mathbf{2}}\right)$ \\
\hline Proliferative phase (7) & $43(32-57)$ & $27.8(22-40.5)$ \\
\hline Secretory phase (9) & $41(21-47)$ & $22.6(18.9-31.6)$ \\
\hline Postmenopausal (7) & $62(52-85)$ & $24.3(20-39.6)$ \\
\hline Total Endometrial cancer pts (24) & $67(37-80)$ & $30(23.9-54.4)$ \\
\hline Endometrioid Grade 1 (6/24) & $61(46-73)$ & $37.8(28.3-46.1)$ \\
\hline Endometrioid Grade 2 (7/24) & $60(37-77)$ & $28.9(25.8-54.4)$ \\
\hline Endometrioid Grade 3 (5/24) & $68(60-80)$ & $29.8(23.9-42.7)$ \\
\hline Malignant Mixed Mullerian Tumor (2/24) & $72.5(65-80)$ & $28.6(24.2-32.9)$ \\
\hline Clear Cell Carcinoma (2/24) & $71.5(61-82)$ & $28.4(26.6-30.1)$ \\
\hline Serous Carcinoma (2/24) & $73(68-78)$ & $32.7\left(\mathrm{NK}{ }^{* *}-32.7\right)$ \\
\hline
\end{tabular}

\subsection{RNA Extraction and Real Time-qPCR}

RNA was extracted from tissue samples using the Trizol Plus RNA extraction kit (Invitrogen, Life Technologies, Paisley, UK) and quantified using Nanodrop ND-1000 (Thermo Fisher Scientific, Loughborough, UK) as previously described [32]. One microgram of RNA was reverse transcribed with random hexamers using AMV reverse transcriptase (New England Biolabs, Hertfordshire, UK). Two micrograms of cDNA were amplified in triplicate for 40 cycles using iTaq universal SYBR Green supermix and CFX Connect Real Time System (Bio-Rad, Hertfordshire, UK). The specific oligonucleotides used to amplify TERRA transcripts from different chromosome ends (Ch 1q-2q-4q-10q-13p-22p, Ch16p, 
Ch 20q) and reaction conditions are listed in Table S1. No template and no reverse transcriptase controls were included on each plate and the melt curves were examined to ensure that only a single product was obtained. Relative transcript level was calculated using the $\triangle \triangle C T$ method, normalized to the reference genes, beta actin $(A C T B)$ and peptidylprolyl Isomerase A (PPIA) using Bio Rad CFX Manager (Bio-Rad, Hertfordshire, UK).

\subsection{Telomerase Repeat Amplification Protocol (TRAP) Assay}

TA was measured using TeloTAGGG TRAP assay (Roche Diagnostics Ltd., Burgess Hill, UK) using $1 \mu \mathrm{g}$ of lysate [22]. Amplification products after 30 cycles were measured as absorbance at $450 \mathrm{~nm}$ in a Fluostar Omega Plate reader (BMG LABTECH, Aylesbury, UK) and presented as arbitrary units (AU).

\subsection{Single Telomere Length Analysis (STELA)}

DNA was extracted using the QIAamp DNA Blood Mini Kit (Qiagen, Manchester, UK). For telomere length analysis at the XpYp telomere, we used the single telomere length analysis (STELA) assay, as previously described [39,44], Genomic DNA was solubilized and diluted in $10 \mathrm{mmol} / \mathrm{L}$ Tric- $\mathrm{HCl}$ ( $\mathrm{pH} 7.5$ ) to $10 \mathrm{ng} / \mu \mathrm{L}$. Ten nanograms of DNA were further diluted with $1 \mu \mathrm{mol} / \mathrm{L}$ Telorette2 linker and $1 \mathrm{mM}$ Tris- $\mathrm{HCl}$ to $250 \mathrm{pg} / \mu \mathrm{L}$ in a volume of $40 \mu \mathrm{L}$. Multiple polymerase chain reactions were conducted to test the DNA sample and were cycled in a Tetrad2 thermocycler (BioRad, Hertfordshire, UK) ( 22 cycles of $94^{\circ} \mathrm{C}$ for $15 \mathrm{~s}, 65^{\circ} \mathrm{C}$ for $30 \mathrm{~s}, 68^{\circ} \mathrm{C}$ for $8 \mathrm{~min}$ ). DNA fragments were resolved by $0.5 \%$ TAE agarose gel electrophoresis and detected by southern hybridization. The hybridized fragments were detected using a phosphorimaging with a Typhoon FLA 9500 phosphoimager (GE healthcare, Chalfont St Giles, UK). The molecular weights of the DNA fragments were calculated using the Phoretix ID quantifier (Nonlinear Dynamics, Newcastle Upon Tyne, UK).

\subsection{Immunohistochemistry}

Formalin-fixed paraffin embedded $3 \mu \mathrm{m}$ tissue sections were immuno-stained with anti-human TRF1, TRF2, steroid receptors and Ki67 antibodies after antigen retrieval at pH6 as previously described [45]. Antibody sources, concentrations and incubation conditions are detailed in Table S2. Matching isotype $(0.5 \mu \mathrm{g} / \mathrm{mL})$ replaced the primary antibody as a negative control. A specific endometrial tissue sample with positive staining was included as the internal positive control with each staining experiment.

Steroid receptor, TRF1 and TRF2, immunostaining was assessed semi-quantitatively using a four-tiered Liverpool endometrial steroid quick score (LESQS) for steroid receptors and a standard quick score for TRF1 and TRF2 as previously described [45]. The Ki67 proliferative index (PI) was evaluated as the percentage of immunopositive cells of any intensity. Epithelial and stromal cell staining was scored separately based on morphological criteria in postmenopausal and malignant endometrium and stratum basalis of healthy proliferative phase and secretory phase endometrium by two independent observers. Discrepancies between the two observers were resolved by re-evaluating the samples together and agreeing on a final score.

\subsection{Analysis of TCGA Dataset}

The publicly available TCGA cohort of uterine cancers included data for RNA levels for TERF1 and was interrogated using Illumina's Base Space Cohort Analyzer application (BSCA) (https://www.illumina. com/informatics/research/biological-data-interpretation/nextbio.html; Illumina, San Diego, CA, USA) [46].

\subsection{Statistical Analysis}

Gene expression data were analyzed using GraphPad Prism software version 5 (San Diego, CA, USA) using non-parametric tests (Mann-Whitney U test or Spearman correlation as appropriate, not-assuming Gaussian distribution). The criterion for significance was $p \leq 0.05$. 


\section{Conclusions}

TERRAs seem to be dynamically expressed in the healthy human endometrium, and significantly lower TERRA levels are found in ECs. Since our study showed TERRA levels to be inversely related to endometrial epithelial cellular proliferative indices, further studies are required to determine whether these levels can be successfully modified and tailored as a therapeutic target in endometrial proliferative conditions.

Supplementary Materials: Supplementary Materials can be found at http://www.mdpi.com/1422-0067/21/22/ 8686/s1. Figure S1: The Cancer Genome Atlas (TCGA) RNA Sequencing Data (endometrioid and serous cancers) for shelterin protein TRF1, Table S1: Oligonucleotides used for qPCR amplification, Table S2: Primary antibodies and conditions for IHC.

Author Contributions: D.K.H. conceived the study and obtained ethical approval. D.K.H., M.A., S.B.D. and J.K. collected samples. M.A., R.E.J., D.M.B., J.D., A.M.K., G.S., R.A., L.B. and S.S. carried out experiments. M.A. and D.K.H. produced the first draft and all authors were involved in revising the paper. All authors have read and agreed to the published version of the manuscript.

Funding: We acknowledge the support by Wellbeing of Women project grants RG1073 and RG1487 (DKH) and Liverpool Women's Hospital Cancer Charity Fund (MA), Liverpool Women's Hospital Foundation Trust (MA, JAD, JK, SBD and DKH), Institute of Translational Medicine (MA, DKH), and Higher Committee for Education Development in Iraq (RA and AMK). The Baird laboratory was supported by Cancer Research UK (C17199/A18246/A29202).

Acknowledgments: The authors would like to thank Annabelle Decottignies of Université Catholique de Louvain for advice on TERRA qPCR optimization. We are grateful to all patients who participated and kindly donated tissue towards research and all staff at Liverpool Women's Hospital and Women's health, Institute of Translational medicine, University of Liverpool who helped for this project.

Conflicts of Interest: The authors declare no conflict of interest. The funders had no role in the design of the study; in the collection, analyses, or interpretation of data; in the writing of the manuscript, or in the decision to publish the results.

\section{References}

1. Lindemann, K.; Eskild, A.; Vatten, L.J.; Bray, F. Endometrial cancer incidence trends in Norway during 1953-2007 and predictions for 2008-2027. Int. J. Cancer 2010, 127, 2661-2668. [CrossRef] [PubMed]

2. CRUK. Available online: https://www.cancerresearchuk.org/health-professional/cancer-statistics/statisticsby-cancer-type/uterine-cancer/mortality (accessed on 3 February 2020).

3. Barcellini, A.; Roccio, M.; Laliscia, C.; Zanellini, F.; Pettinato, D.; Valvo, F.; Mirandola, A.; Orlandi, E.; Gadducci, A. Endometrial Cancer: When Upfront Surgery Is Not an Option. Oncology 2020, 1-7. [CrossRef] [PubMed]

4. Hapangama, D.K.; Kamal, A.; Saretzki, G. Implications of telomeres and telomerase in endometrial pathology. Hum. Reprod. Update 2017, 23, 166-187. [CrossRef] [PubMed]

5. Alnafakh, R.A.A.; Adishesh, M.; Button, L.; Saretzki, G.; Hapangama, D.K. Telomerase and Telomeres in Endometrial Cancer. Front. Oncol. 2019, 9, 344. [CrossRef]

6. Smogorzewska, A.; de Lange, T. Regulation of telomerase by telomeric proteins. Annu. Rev. Biochem. 2004, 73, 177-208. [CrossRef]

7. Kim, N.W.; Piatyszek, M.A.; Prowse, K.R.; Harley, C.B.; West, M.D.; Ho, P.L.; Coviello, G.M.; Wright, W.E.; Weinrich, S.L.; Shay, J.W. Specific association of human telomerase activity with immortal cells and cancer. Science 1994, 266, 2011-2015. [CrossRef]

8. Nergadze, S.G.; Farnung, B.O.; Wischnewski, H.; Khoriauli, L.; Vitelli, V.; Chawla, R.; Giulotto, E.; Azzalin, C.M. CpG-island promoters drive transcription of human telomeres. RNA 2009, 15, 2186-2194. [CrossRef]

9. Azzalin, C.M.; Reichenbach, P.; Khoriauli, L.; Giulotto, E.; Lingner, J. Telomeric repeat containing RNA and RNA surveillance factors at mammalian chromosome ends. Science 2007, 318, 798-801. [CrossRef]

10. Luke, B.; Panza, A.; Redon, S.; Iglesias, N.; Li, Z.; Lingner, J. The Rat1p 5' to $3^{\prime}$ exonuclease degrades telomeric repeat-containing RNA and promotes telomere elongation in Saccharomyces cerevisiae. Mol. Cell 2008, 32, 465-477. [CrossRef] 
11. Vrbsky, J.; Akimcheva, S.; Watson, J.M.; Turner, T.L.; Daxinger, L.; Vyskot, B.; Aufsatz, W.; Riha, K. siRNA-mediated methylation of Arabidopsis telomeres. PLoS Genet. 2010, 6, e1000986. [CrossRef]

12. Beishline, K.; Vladimirova, O.; Tutton, S.; Wang, Z.; Deng, Z.; Lieberman, P.M. CTCF driven TERRA transcription facilitates completion of telomere DNA replication. Nat. Commun. 2017, 8, 2114. [CrossRef] [PubMed]

13. Montero, J.J.; López de Silanes, I.; Graña, O.; Blasco, M.A. Telomeric RNAs are essential to maintain telomeres. Nat. Commun. 2016, 7, 12534. [CrossRef] [PubMed]

14. Arnoult, N.; Van Beneden, A.; Decottignies, A. Telomere length regulates TERRA levels through increased trimethylation of telomeric H3K9 and HP1 $\alpha$. Nat. Struct. Mol. Biol. 2012, 19, 948-956. [CrossRef] [PubMed]

15. Smekalova, E.; Baumann, P. TERRA -a calling card for telomerase. Mol. Cell 2013, 51, 703-704. [CrossRef]

16. Hirashima, K.; Seimiya, H. Telomeric repeat-containing RNA/G-quadruplex-forming sequences cause genome-wide alteration of gene expression in human cancer cells in vivo. Nucleic Acids Res. 2015, 43, 2022-2032. [CrossRef]

17. Graf, M.; Bonetti, D.; Lockhart, A.; Serhal, K.; Kellner, V.; Maicher, A.; Jolivet, P.; Teixeira, M.T.; Luke, B. Telomere Length Determines TERRA and R-Loop Regulation through the Cell Cycle. Cell 2017, 170, 72-85.e14. [CrossRef]

18. Redon, S.; Reichenbach, P.; Lingner, J. The non-coding RNA TERRA is a natural ligand and direct inhibitor of human telomerase. Nucleic Acids Res. 2010, 38, 5797-5806. [CrossRef]

19. Porro, A.; Feuerhahn, S.; Reichenbach, P.; Lingner, J. Molecular dissection of telomeric repeat-containing RNA biogenesis unveils the presence of distinct and multiple regulatory pathways. Mol. Cell. Biol. 2010, 30, 4808-4817. [CrossRef]

20. Marion, R.M.; Montero, J.J.; Lopez de Silanes, I.; Grana-Castro, O.; Martinez, P.; Schoeftner, S.; Palacios-Fabrega, J.A.; Blasco, M.A. TERRA regulate the transcriptional landscape of pluripotent cells through TRF1-dependent recruitment of PRC2. eLife 2019, 8. [CrossRef]

21. Hapangama, D.K.; Bulmer, J.N. Pathophysiology of heavy menstrual bleeding. Womens Health (Lond.) 2016, 12, 3-13. [CrossRef]

22. Valentijn, A.J.; Saretzki, G.; Tempest, N.; Critchley, H.O.; Hapangama, D.K. Human endometrial epithelial telomerase is important for epithelial proliferation and glandular formation with potential implications in endometriosis. Hum. Reprod. 2015, 30, 2816-2828. [CrossRef] [PubMed]

23. Menon, M.M.; Simha, M.R. Telomerase and telomere length in normal and malignant human endometrium as prognostic markers. Indian J. Pathol. Microbiol. 2003, 46, 394-398. [PubMed]

24. Saygan-Karamursel, B.; Dikmen, G.; Dogan, P.; Aksu, T.; Guven, S.; Ayhan, A. Quantitative telomerase activity in malignant, benign and normal gynecological tissues. Eur. J. Gynaecol. Oncol. 2005, 26, 83-86. [PubMed]

25. Bradfield, A.; Button, L.; Drury, J.; Green, D.C.; Hill, C.J.; Hapangama, D.K. Investigating the Role of Telomere and Telomerase Associated Genes and Proteins in Endometrial Cancer. Methods Protoc. 2020, 3. [CrossRef] [PubMed]

26. Williams, C.D.; Boggess, J.F.; LaMarque, L.R.; Meyer, W.R.; Murray, M.J.; Fritz, M.A.; Lessey, B.A. A prospective, randomized study of endometrial telomerase during the menstrual cycle. J. Clin. Endocrinol. Metab. 2001, 86, 3912-3917. [CrossRef]

27. Tanaka, M.; Kyo, S.; Takakura, M.; Kanaya, T.; Sagawa, T.; Yamashita, K.; Okada, Y.; Hiyama, E.; Inoue, M. Expression of telomerase activity in human endometrium is localized to epithelial glandular cells and regulated in a menstrual phase-dependent manner correlated with cell proliferation. Am. J. Pathol. 1998, 153, 1985-1991. [CrossRef]

28. Wang, S.-J.; Sakamoto, T.; Yasuda, S.-i.; Fukasawa, I.; Ota, Y.; Hayashi, M.; Okura, T.; Zheng, J.-H.; Inaba, N. The Relationship between Telomere Length and Telomerase Activity in Gynecologic Cancers. Gynecol. Oncol. 2002, 84, 81-84. [CrossRef]

29. Kyo, S.; Kanaya, T.; Ishikawa, H.; Ueno, H.; Inoue, M. Telomerase activity in gynecological tumors. Clin. Cancer Res. 1996, 2, 2023-2028.

30. Kyo, S.; Takakura, M.; Kohama, T.; Inoue, M. Telomerase activity in human endometrium. Cancer Res. 1997, 57, 610-614.

31. Shay, J.W. Role of Telomeres and Telomerase in Aging and Cancer. Cancer Discov. 2016, 6, 584-593. [CrossRef] 
32. Mathew, D.; Drury, J.A.; Valentijn, A.J.; Vasieva, O.; Hapangama, D.K. In silico, in vitro and in vivo analysis identifies a potential role for steroid hormone regulation of FOXD3 in endometriosis-associated genes. Hum. Reprod. 2016, 31, 345-354. [CrossRef] [PubMed]

33. Maestroni, L.; Audry, J.; Matmati, S.; Arcangioli, B.; Geli, V.; Coulon, S. Eroded telomeres are rearranged in quiescent fission yeast cells through duplications of subtelomeric sequences. Nat. Commun. 2017, 8, 1684. [CrossRef] [PubMed]

34. van Steensel, B.; de Lange, T. Control of telomere length by the human telomeric protein TRF1. Nature 1997, 385, 740-743. [CrossRef] [PubMed]

35. Schoeftner, S.; Blasco, M.A. Developmentally regulated transcription of mammalian telomeres by DNA-dependent RNA polymerase II. Nat. Cell Biol. 2008, 10, 228-236. [CrossRef] [PubMed]

36. Deng, Z.; Norseen, J.; Wiedmer, A.; Riethman, H.; Lieberman, P.M. TERRA RNA binding to TRF2 facilitates heterochromatin formation and ORC recruitment at telomeres. Mol. Cell 2009, 35, 403-413. [CrossRef]

37. Hu, Y.; Bennett, H.W.; Liu, N.; Moravec, M.; Williams, J.F.; Azzalin, C.M.; King, M.C. RNA-DNA Hybrids Support Recombination-Based Telomere Maintenance in Fission Yeast. Genetics 2019, 213, 431-447. [CrossRef]

38. Barthel, F.P.; Wei, W.; Tang, M.; Martinez-Ledesma, E.; Hu, X.; Amin, S.B.; Akdemir, K.C.; Seth, S.; Song, X.; Wang, Q.; et al. Systematic analysis of telomere length and somatic alterations in 31 cancer types. Nat. Genet. 2017, 49, 349-357. [CrossRef]

39. Baird, D.M.; Rowson, J.; Wynford-Thomas, D.; Kipling, D. Extensive allelic variation and ultrashort telomeres in senescent human cells. Nat. Genet. 2003, 33, 203-207. [CrossRef]

40. Wang, C.; Zhao, L.; Lu, S. Role of TERRA in the regulation of telomere length. Int. J. Biol. Sci. 2015, 11, 316-323. [CrossRef]

41. Cusanelli, E.; Romero, C.A.; Chartrand, P. Telomeric noncoding RNA TERRA is induced by telomere shortening to nucleate telomerase molecules at short telomeres. Mol. Cell 2013, 51, 780-791. [CrossRef]

42. Takahama, K.; Takada, A.; Tada, S.; Shimizu, M.; Sayama, K.; Kurokawa, R.; Oyoshi, T. Regulation of telomere length by G-quadruplex telomere DNA- and TERRA-binding protein TLS/FUS. Chem. Biol. 2013, 20, 341-350. [CrossRef] [PubMed]

43. Porro, A.; Feuerhahn, S.; Lingner, J. TERRA-reinforced association of LSD1 with MRE11 promotes processing of uncapped telomeres. Cell Rep. 2014, 6, 765-776. [CrossRef] [PubMed]

44. Lin, T.T.; Letsolo, B.T.; Jones, R.E.; Rowson, J.; Pratt, G.; Hewamana, S.; Fegan, C.; Pepper, C.; Baird, D.M. Telomere dysfunction and fusion during the progression of chronic lymphocytic leukemia: Evidence for a telomere crisis. Blood 2010, 116, 1899-1907. [CrossRef]

45. Kamal, A.M.; Bulmer, J.N.; DeCruze, S.B.; Stringfellow, H.F.; Martin-Hirsch, P.; Hapangama, D.K. Androgen receptors are acquired by healthy postmenopausal endometrial epithelium and their subsequent loss in endometrial cancer is associated with poor survival. Br. J. Cancer 2016, 114, 688-696. [CrossRef] [PubMed]

46. Kupershmidt, I.; Su, Q.J.; Grewal, A.; Sundaresh, S.; Halperin, I.; Flynn, J.; Shekar, M.; Wang, H.; Park, J.; Cui, W.; et al. Ontology-based meta-analysis of global collections of high-throughput public data. PLoS ONE 2010, 5, e13066. [CrossRef]

Publisher's Note: MDPI stays neutral with regard to jurisdictional claims in published maps and institutional affiliations.

(C) 2020 by the authors. Licensee MDPI, Basel, Switzerland. This article is an open access article distributed under the terms and conditions of the Creative Commons Attribution (CC BY) license (http://creativecommons.org/licenses/by/4.0/). 